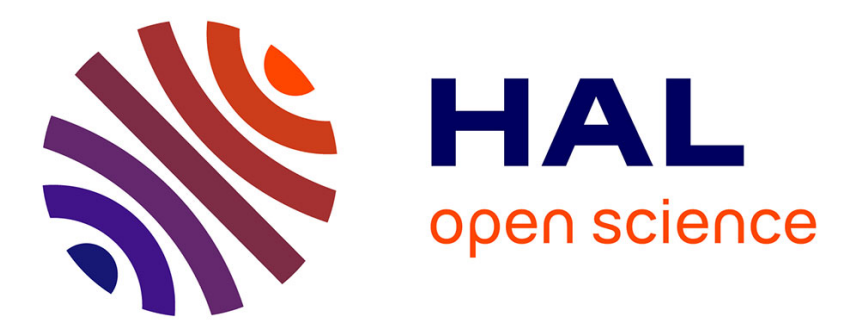

\title{
Inhibition of phase turbulence close to onset of convection by permeable lateral boundary condition for the mean flow
}

\author{
F. Daviaud, A Pocheau
}

\section{- To cite this version:}

F. Daviaud, A Pocheau. Inhibition of phase turbulence close to onset of convection by permeable lateral boundary condition for the mean flow. EPL - Europhysics Letters, 1989, 9 (7), pp.675-680. cea-01374041

\section{HAL Id: cea-01374041 https://hal-cea.archives-ouvertes.fr/cea-01374041}

Submitted on 29 Sep 2016

HAL is a multi-disciplinary open access archive for the deposit and dissemination of scientific research documents, whether they are published or not. The documents may come from teaching and research institutions in France or abroad, or from public or private research centers.
L'archive ouverte pluridisciplinaire $\mathbf{H A L}$, est destinée au dépôt et à la diffusion de documents scientifiques de niveau recherche, publiés ou non, émanant des établissements d'enseignement et de recherche français ou étrangers, des laboratoires publics ou privés. 


\section{HAL \\ archives-ouvertes.}

\section{Inhibition of phase turbulence close to onset of convection by permeable lateral boundary condition for the mean flow}

F. Daviaud, A Pocheau

\section{- To cite this version:}

F. Daviaud, A Pocheau. Inhibition of phase turbulence close to onset of convection by permeable lateral boundary condition for the mean flow. Europhys. Lett, 1989. <cea-01374041>

\section{HAL Id: cea-01374041 \\ https://hal-cea.archives-ouvertes.fr/cea-01374041}

Submitted on 29 Sep 2016

HAL is a multi-disciplinary open access archive for the deposit and dissemination of scientific research documents, whether they are published or not. The documents may come from teaching and research institutions in France or abroad, or from public or private research centers.
L'archive ouverte pluridisciplinaire HAL, est destinée au dépôt et à la diffusion de documents scientifiques de niveau recherche, publiés ou non, émanant des établissements d'enseignement et de recherche français ou étrangers, des laboratoires publics ou privés. 
Europhys. Lett., 9 (7), pp. 675-680 (1989)

\title{
Inhibition of Phase Turbulence Close to Onset of Convection by Permeable Lateral Boundary Condition for the Mean Flow.
}

\author{
F. Daviaud $\left(^{*}\right)$ and A. Pocheau (**) \\ (*) Institut de Recherche Fondamentale, DPh.G, SRM \\ CEA Orme des Merisiers, 91191 Gif-sur-Yvette, France \\ (**) Laboratoire de Recherche en Combustion, Université de Provence \\ Centre de Saint-Jérome, S252-13397 Marseille, France
}

(received 23 January 1989; accepted in final form 23 May 1989)

PACS. 47.25 - Turbulent flows, convection, and heat transfer.

PACS. 47.25Q - Convection and heat transfer.

PACS. 47.20 - Hydrodynamic stability and instability.

\begin{abstract}
We show that the mechanisms which govern the onset of time dependence in usual Rayleigh-Bénard convection at low Prandtl number may be inhibited by a suitable choice of the lateral boundary condition for the sole mean flow. We first build a boundary condition which behaves like a rigid one for the roll flow and like a permeable one for the mean flow. We then observe that phase turbulence is inhibited close to the onset of convection. We understand this effect by solving the mean-flow field from the Cross-Newell equations. Our experimental result together with its interpretation demonstrates indirectly the existence of mean flows and enlightens the ways by which mean flows destabilize patterns.
\end{abstract}

In the past decade, Rayleigh-Bénard convection in extended systems has brought about a puzzling paradox: at low Prandtl number $(P r=0.7)$, the threshold of time dependence is roughly one order of magnitude lower for distorted patterns $(\varepsilon=O(0.1))[1,2]$ than for straight ones $(\varepsilon \approx 3)[3]\left(\varepsilon=\left[R a-R a_{\mathrm{c}}\right] / R a_{\mathrm{c}}\right.$, where $R a$ is the Rayleigh number and $R a_{\mathrm{c}}$ its value at the onset of convection). Thus pattern distortion represents quite a dangerous mode, capable of leading to an overdestabilization. The origin of this mechanism has been traced back to the existence of large scale parallel flows (i.e. mean flows) which are produced by the roll flows, whenever the roll structure is distorted [4]. It has already been proved that large-scale flows play a crucial role in the instabilities of straight roll patterns [5] and in the transition to both time dependence and turbulence in a cylindrical container [6-9]. In this letter, we aim at showing that the spatio-temporal properties of convective patterns, including their route to turbulence, may be changed by suitable action on the sole large-scale flow. In particular, we have been able to inhibit phase turbulence close to onset of convection in a cylindrical container and thus to obtain stationary distorted patterns at values of $\varepsilon$ one order of magnitude greater than in usual configurations. This result proves indirectly the existence of mean flows and enlightens the mechanisms by which pattern overdestabilization arises. 
Let us first recall the mechanisms of time dependence in usual convection experiments, i.e. in closed containers. For cylindrical geometries and at $\mathrm{Pr}=0.7$, visual observations show that rolls tend to end perpendicularly to the walls so that two centres of pattern curvature arise near the onset of convection (similarly to fig. 1a)). Roll curvature then
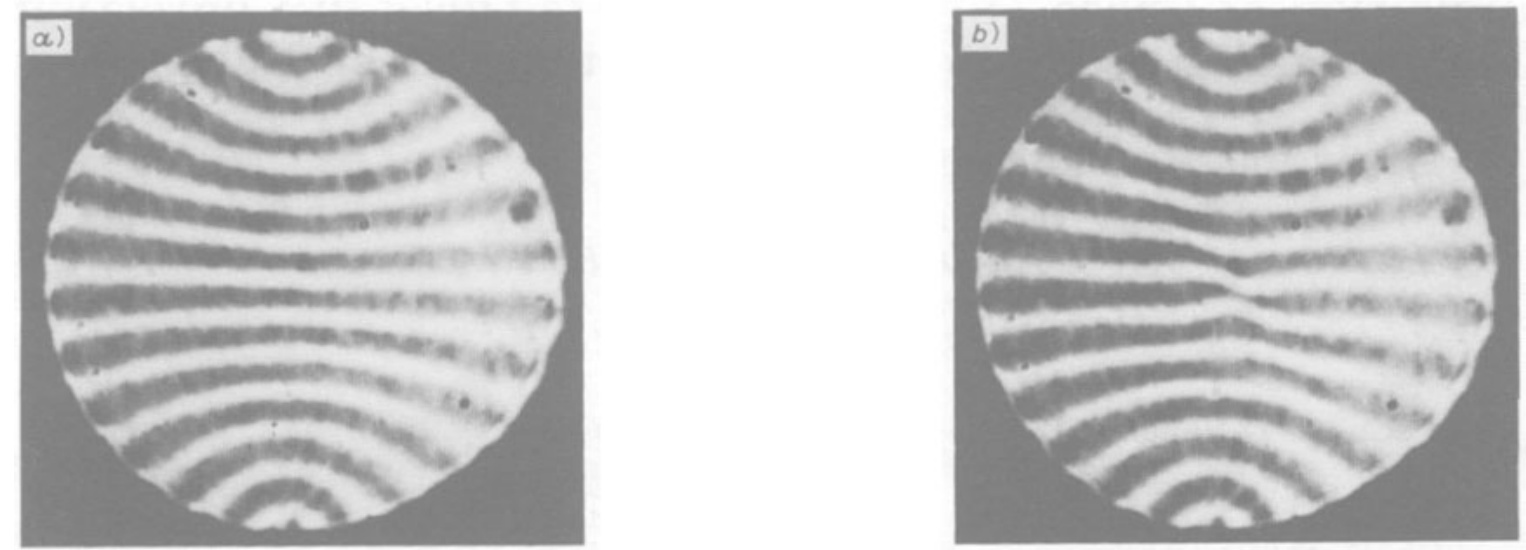

Fig. 1. - Pictures of the open container. a) Stationary pattern at $\varepsilon=0.55$. The $y$-axis is defined such that it joins the foci. $b$ ) Occurrence of the first instability at $\varepsilon=0.56$. Notice the skewed-varicose-like distortion.

produces mean flows which in turn give rise to roll compression and finally, for $\varepsilon>\varepsilon_{0} \approx 0.1$, to localized instabilities at the centre of the pattern (nucleations of dislocations) and to time dependence [2]. In agreement with numerical simulations [7], we have observed that the threshold of time dependence, $\varepsilon_{0}$, slightly decreases with the aspect ratio $R: \varepsilon_{0}(7.66)=1.4$, $\varepsilon_{0}(12.5)=0.08$ and $\varepsilon_{0}(16)=0.06$, where $R$ is defined as the ratio of the radius of the cylindrical cell to its depth. In this system, mean flows and roll distortion are coupled in a nonlocal way: roll distortion produces mean flows but mean flows may in turn produce distortion, as demonstrated by the study of forced mean flow effects on a chain of rolls [10]. Nonlocality is a consequence of mass conservation (any distorted roll of the convective field participates in producing a mean flow at a given point) and of the scale difference (mean-flow effects accumulate from roll to roll). This nonlocal coupling is described by the Cross-Newell equations [11]. An exact perturbative solution of these equations has been found in a closed cylindrical container for patterns displaying two opposite bendings [8]. In this solution, a back-mean flow is then generated by a pressure gradient on the line joining the foci to the centre of the pattern ( $y$-axis) (fig. $2 a)$ ). In agreement with experiments, it sustains dangerous compression modes in the phase equation which finally trigger roll pinchings by localized instabilities at $\varepsilon=O(0.1)$. We emphasize that the main modes leading to time dependence are roll curvature for producing mean flow and the back flow for yielding overdestabilization. We finally remark that the back flow is a direct consequence of the lateral boundary conditions.

In our experiment, we aim at modifying this mechanism of time dependence by acting on the sole large scale flow independently of the underlying roll structure. To achieve this, we alter the lateral boundary condition for the mean flow, but we keep that for the roll flow unchanged. We therefore expect the rolls to still end perpendicularly to the boundary so that curvature is maintained. As described below, our new boundary stops convection but is permeable to mean flows. We therefore expect the pressure gradient and thus the back flow together with the route to time dependence, to be modified. 

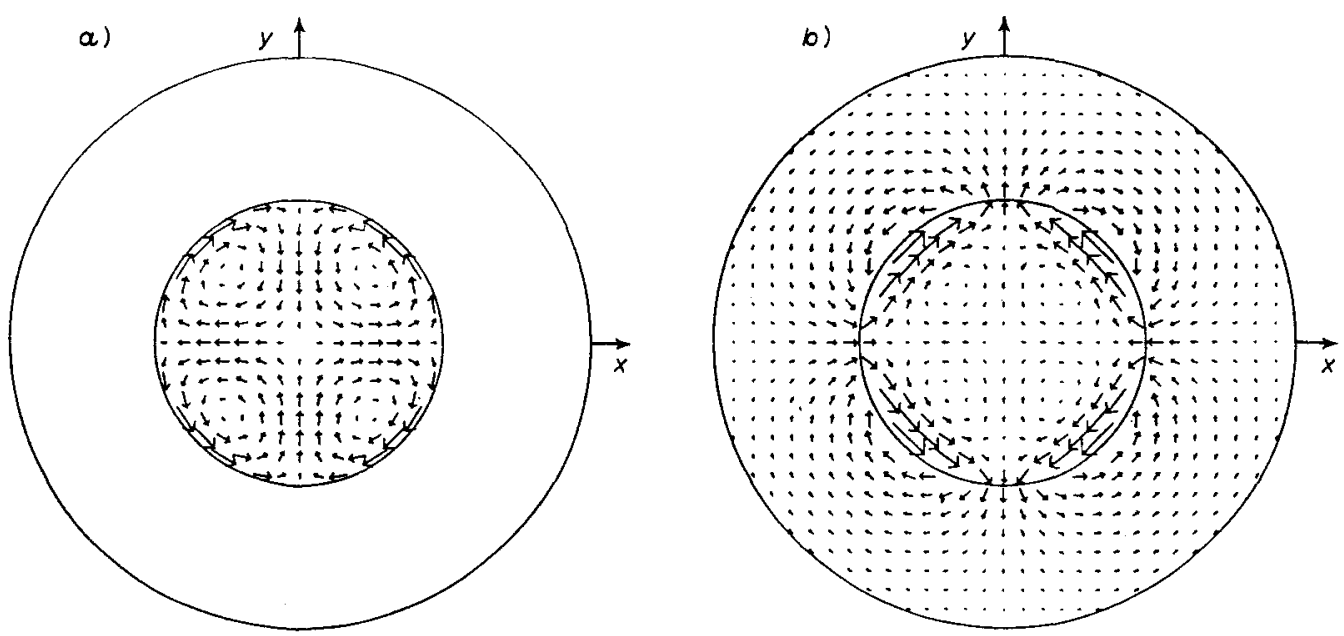

Fig. 2. - Mean-flow field solutions of the Cross-Newell equations. a) The boundary of the convective domain (small circle) is nonpermeable to mean flows. Notice the back flows along the $y$-axis (the orientation is defined in fig. 1a)). b) The boundary of the convective domain is permeable to mean flows. The mean flow amplitude is then weaker than in the closed configuration. The shear at the boundary of the convective domain generates localized additional vertical vorticity, not represented on the picture.

Since mean flows are generated in a nonlocal way by the whole pattern, inhibiting convection outside a given domain should not stop them, provided the boundaries are not rigid walls. In order to satisfy this condition, we slightly lower the cell depth outside a cylindrical domain by inserting a thin sheet at quarter of the cell height (fig. 3 ). This trick gives rise to negligible thermal perturbations but to strong flow modifications: only conduction can take place in the outer region and the sheet is expected to let a large part of the mean flows pass. Visual observations ensure that the sheet is sufficient to give rise to roll curvature. Finally, as in usual cells, lateral walls protect from external thermal or flow perturbations.

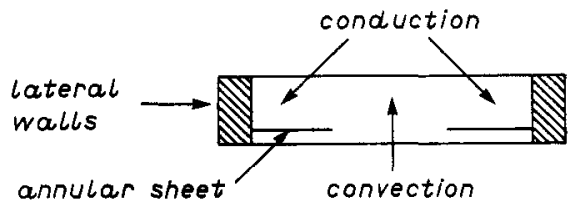

Fig. 3. - Sketch of the experimental set-up leading to a permeable lateral boundary condition for the mean flows.

We work in argon gas under a pressure of 30 bars $(\operatorname{Pr}=0.7)$. We use an experimental apparatus similar to that described in [2], the extension of the visual field having been increased, however. The walls are cylindrical and the cell aspect ratio is $R^{\prime}=25$. The cell depth is $1.6 \mathrm{~mm}$ so that the critical temperature is $3.5^{\circ} \mathrm{C}$. The thin sheet has an annular geometry. It gives rise to a cylindrical convective region of aspect ratio $R=12.5$ (fig. 3).

At onset of convection, rolls are straight. As $\varepsilon$ is increased, two centres of curvature appear and the curvature grows but saturates at $\varepsilon \approx 0.2$. This is in agreement with observations in a closed container, except that no dangerous compression occurs at the 
centre of the pattern. Moreover, the pattern remains stationary up to surprisingly high values of $\varepsilon$ (fig. 1a)). Such stationary states are reached after transient evolution, whatever the initial condition is. Sustained time dependence occurs only at $\varepsilon=1.2$ and involves repetitive localized processes which are reminiscent of the skewed-varicose and cross-roll instabilities of Busse and Clever [12]. The threshold of time dependence of distorted patterns is thus one order of magnitude greater in an "open container» than in a closed one. Time dependence and phase turbulence are therefore inhibited over a large range of $\varepsilon$.

The system undergoes several restabilization processes towards stationary patterns in its route to turbulence. The first process leads to a wave number selection. It arises at $\varepsilon=0.56$ by a roll pinching at the centre of the pattern (fig. $1 b$ )) which leads to the nucleation of a pair of dislocations which eventually disappear at the foci. The whole pattern has then lost one wavelength. It remains stationary up to $\varepsilon=0.74$, where a new roll pinching occurs at the centre of the pattern. Further additional defect nucleations yield a long transient spatio-temporal evolution which relaxes towards a complex stationary pattern. Stationarity persists until $\varepsilon=1.2$, where localized phase oscillations occur by periodic cross-roll-like instabilities or grain boundary motions. Finally, at $\varepsilon=1.5$, observations on a time scale of the order $R \cdot \tau_{\mathrm{h}}$, where $\tau_{\mathrm{h}}$ is the horizontal diffusion time, show phase turbulence. Focus singularities near the boundary then display phase travelling waves which either generate new rolls or force rolls to disappear. The latter feature is never observed in a closed container, presumably because mean flows cannot escape the convective domain.

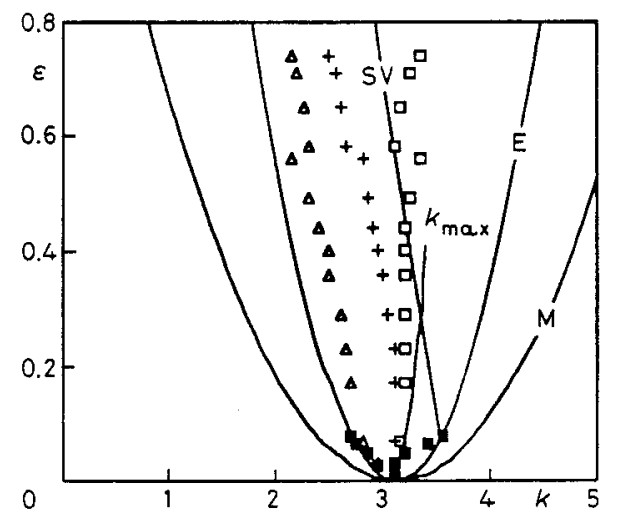

Fig. 4. - Stability diagram of infinite straight rolls at $P r=0.7$ displaying the marginal (M), the Eckhaus (E) and the skewed-varicose (SV) stability curves. We have plotted the local wave numbers for closed and open containers. Black squares correspond to the band of wave numbers in a closed container and triangles, crosses and open squares to the wave numbers in an open container, respectively at the boundary on the $x$-axis, the focus and the centre. The line $\left(k_{\max }\right)$ corresponds to the maximum wave number of the solution of the Cross-Newell equations, the sheet viscous stress being taken into account.

Measurements of the roll wave number distribution allow us to compare quantitatively the pattern behaviour in closed and open cells. As shown in fig. 4, the first two dislocation nucleations occur at a local wave number which, as in a closed container, is unstable with respect to the skewed-varicose instability, the shift with the marginal stability being probably due to finite-size effects produced by the localization. However, this local wavenumber crosses the straight roll stability domain at values of $\varepsilon$ five times greater in open cells than in closed cells. This corroborates the observation of a delay in the occurrence of time dependence. In addition, we note that the decrease of the wavenumber allows the 
restabilization of patterns. For both these reasons, although the pattern is distorted, its behaviour is similar to that of straight rolls [3].

Moreover, in contrast with the case of closed containers, the wave numbers at the foci and at the centre of the pattern have quite similar values. This means that the compression of the structure has been largely reduced, so that no dangerous large wave number is encountered close to the convective threshold. This indicates that, as expected, the meanflow field has been modified by the change of lateral boundary in such a way that it does not induce destabilizing distortions. In particular, our measurements evidence that the amplitude of the back flow is strongly lowered.

In order to corroborate these experimental results, we have determined [13] the meanflow field by expansion of the Cross-Newell equations in a way similar to that performed in a closed container [8]. The phase field $\varphi$ is thus modelled in a polynomial space $\phi(x, y)=$ $=k_{0}(1+\Delta) y\left(1-a x^{2} / R^{2}+b y^{2} / R^{2}+c y^{4} / R^{4}+d x^{2} y^{2} / R^{2}\right)$ where the relation between $(b, c, d, \Delta)$ and $a$ has to be found by expansion at first order in $a^{2}$ and $1 / R$, assuming $R \gg 1$ and $a \ll 1$ and where $k_{0}$ is close to $k_{\mathrm{c}}=3.117$ at $\mathrm{Pr}=0.7$. In a closed container, we recall that the mean-flow vertical vorticity reads $\Omega_{z}=2 \omega x y / R^{4}$ ( $\omega$ is positive and roughly inversely proportional to the Prandtl number). Moreover, the orientation being defined such that the $y$-axis $(\theta= \pm \pi / 2)$ joins the foci (fig. 1a)), the mean-flow field $\boldsymbol{F}$ takes the following form in polar coordinates:

$$
\boldsymbol{F}=\frac{\omega}{6 R^{4}}\left[r\left(r^{2}+\beta R^{2}\right)\left|\begin{array}{c}
-\cos (2 \theta) \\
\sin (2 \theta)
\end{array}\right|+r^{3}\left|\begin{array}{c}
0 \\
\sin (2 \theta)
\end{array}\right|\right]+o\left(\frac{a^{2}}{R}\right),
$$

where $\beta$ is only related to a divergence-free pressure gradient.

Since the container is closed, the normal flow $\boldsymbol{F} \cdot \boldsymbol{r}$ must vanish at the boundary $r=R$, so that $\beta=-1$. In an «open» container, we consider, as a first step, that mean flows cross the boundary of the convective domain quite freely. In order to determine the mean-flow field in both the conductive and convective domains, we first assume that no additional source of large-scale vertical vorticity $\Omega_{z}$ is generated by the new boundary. The boundary conditions therefore involve the continuity at $r=R$ of the pressure, the vorticity $\Omega_{z}$ and the radial flux $\boldsymbol{F} \cdot \boldsymbol{r}$ and the vanishing at $r=R^{\prime}$ of the radial flux. In the convective domain, $\Omega_{z}$ is similar to that of a closed container but it must diffuse and vanish in the conductive domain, since there are no longer mean-flow sources there. For large $R$, we find that the mean-flow field takes the previous form (1) inside the convective domain with $\beta=-1 / 2\left(1+\rho^{-4}\right)$ and $\rho=R^{\prime} / R$, and the following form in the conductive region [13]:

$$
\boldsymbol{F}=-\frac{\omega}{12} \frac{R^{2}}{r^{3}}\left|\begin{array}{l}
\cos (2 \theta)\left[1-\frac{r^{4}}{R^{\prime 4}}\right] \\
\sin (2 \theta)\left[1+\frac{r^{4}}{R^{\prime 4}}\right]
\end{array}\right|+o\left(\frac{a^{2}}{R}\right)
$$

As shown in fig. $2 b$ ), the mean-flow streamlines are strongly modified by the change of lateral boundary. In particular, a shear flow appears at the boundary of the convective domain so that additional vorticity is created. However, since it remains localized, it does not modify the former determination away from the boundary of the convective domain. Instead of the strong back flow present in closed containers, the mean flow reverses its direction on the line joining a focus to the centre of the pattern and its amplitude is now weaker. Introducing its value in the phase equation enables us to determine the stationary conditions on the large scale. Compared to a closed container [8], the relation between $(b, c, d, \Delta)$ and $a$ is unchanged except that $b$ is multiplied by $-\beta$, i.e. $1 / 2\left(1+f^{-4}\right)$. For small 
$e^{-4}\left(f^{-4} \ll 1\right)$, the maximum wave number is then $k_{0}+o\left(a^{2} / R\right)$ and it is reached at both the foci and the centre of the pattern. The latter prediction is close to the experimental results (fig. 4). However, the additional viscous stress produced by the sheet in the conductive domain produces an additional back flow which may be taken into account by introducing a transmission factor. The agreement with the experiment is then even better (fig. 4).

Our solution of the Cross-Newell equations thus nicely recovers the experimental results and agrees with their interpretation. In particular, it confirms that the back flow is weakened. Accordingly, though mean flows are still present, the conditions leading to «dynamical frustration» [6] are less fulfilled than in a closed container. This explains why time dependence is delayed.

We have demonstrated that opening the container to flows other than the primary convective flows changes the spatio-temporal properties of an extended cylindrical convective layer and inhibits phase turbulence in a large range of the control parameter $\varepsilon$. We have solved the Cross-Newell equations for this situation at the dominant order in the amplitude of the pattern distortion. The mean-flow field of this solution enables us to understand the delay observed in the appearance of time dependence. These results prove indirectly the existence of mean flows and confirm the mechanisms of phase turbulence based on the mean flow-roll distortion interaction. In particular, they point out the nonlocal link between mean flows and boundary conditions. At a more general level, our study shows, as an example, that spatio-temporal turbulence may be inhibited, provided that the right phenomena are understood. We envision to extend this control of turbulence to other cellular patterns and to systems exhibiting interactions between more than two scales.

$* * *$

We wish to acknowledge P. Pelcé, P. Bergé, P. Clavin, P. Manneville, H. Chaté, M. Dubois for discussions and M. LABOuISE and B. Ozenda for technical assistance.

\section{REFERENCES}

[1] Ahlers G. and Behringer R. P., Phys. Rev. Lett., 40 (1978) 712.

[2] Pocheau A., Croquette V. and Le Gal P., Phys. Rev. Lett., 55 (1985) 1094.

[3] Kolodner P., Walden R. W., Passner A. and Surko C. M., J. Fluid Mech., 163 (1986) 195; Motsay R. W., ANderson K. E. and Behringer R. P., J. Fluid Mech. 189 (1988) 26; Croquette V., to appear in Contemp. Phys.

[4] Siggia E. D. and ZipPelius A., Phys. Rev. Lett., 47 (1981) 835.

[5] Cross M. C., Phys. Rev. A, 27 (1983) 490; Manneville P. and Piquemal J. M., Phys. Rev. A, 28 (1983) 1774.

[6] Manneville P., J. Phys. Lett., 44 (1983) 903.

[7] Greenside H. S., Cross M. C. and Coughran W. M. jr., Phys. Rev. Lett., 60 (1988) 2269.

[8] Pocheau A., C.R. Acad. Sci., 306 (1988) 331; J. Phys. France, 49 (1988) 1127.

[9] Pocheau A., J. Phys. France, in press.

[10] Pocheau A., Croquette V., Le Gal P. and Poirrou C., Europhys. Lett., 3 (1987) 915; due to a typographic error, the modified phase equation does not contain the mean-flow amplitude $U$ : Erratum, Europhys Lett. 4 (1987) 253.

[11] Cross M. C. and Newell A. C., Physica D, 10 (1984) 299.

[12] Busse F. H. and Clever R. M., J. Fluid Mech., 91 (1979) 319.

[13] Pocheau A. and Daviaud F., to be submitted to Phys. Rev. A. 